\title{
Immune-Enhancing Effect of Nanometric Lactobacillus plantarum $n F 1$ (nLp-nF1) in a Mouse Model of Cyclophosphamide-Induced Immunosuppression
}

\author{
Dae-Woon Choi ${ }^{1,2}$, Sun Young Jung ${ }^{1,2}$, Jisu Kang ${ }^{1,2}$, Young-Do Nam ${ }^{1,2}$, Seong-Il Lim $^{3}$, Ki Tae Kim ${ }^{4}$, and \\ Hee Soon Shin ${ }^{1,2 *}$ \\ ${ }^{1}$ Food Biotechnology Program, Korea University of Science and Technology, Daejeon 34113, Republic of Korea \\ ${ }^{2}$ Division of Nutrition and Metabolism Research, Korea Food Research Institute, Wanju 55365, Republic of Korea \\ ${ }^{3}$ Division of Strategic Food Research, Korea Food Research Institute, Wanju 55365, Republic of Korea \\ ${ }^{4}$ Biogenics Korea Co. Ltd., Seoul 06628, Republic of Korea
}

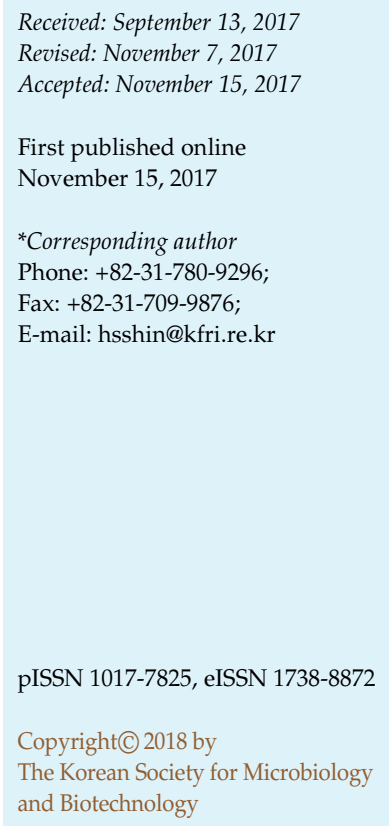

Nanometric Lactobacillus plantarum nF1 (nLp-nF1) is a biogenics consisting of dead L. plantarum cells pretreated with heat and a nanodispersion process. In this study, we investigated the immune-enhancing effects of $n L p-n F 1$ in vivo and in vitro. To evaluate the immunostimulatory effects of nLp-nF1, mice immunosuppressed by cyclophosphamide (CPP) treatment were administered with $n L p-n F 1$. As expected, CPP restricted the immune response of mice, whereas oral administration of $n L p-n F 1$ significantly increased the total IgG in the serum, and cytokine production (interleukin-12 (IL-12) and tumor necrosis factor alpha (TNF- $\alpha$ )) in bone marrow cells. Furthermore, nLp-nF1 enhanced the production of splenic cytokines such as IL-12, TNF- $\alpha$, and interferon gamma (IFN- $\gamma$ ). In vitro, nLp-nF1 stimulated the immune response by enhancing the production of cytokines such as IL-12, TNF- $\alpha$, and IFN- $\gamma$. Moreover, nLp-nF1 given a food additive enhanced the immune responses when combined with various food materials in vitro. These results suggest that nLp-nF1 could be used to strengthen the immune system and recover normal immunity in people with a weak immune system, such as children, the elderly, and patients.

Keywords: Nanometric Lactobacillus plantarum nF1, immune enhancement, interleukin-12, macrophage, immunoglobulin G, cyclophosphamide

\section{Introduction}

The recent years have seen an increase in various infectious disorders, including respiratory diseases, due to rapid industrial development, environmental pollution, and climate changes $[1,2]$. To overcome these health problems, effective vaccines have been developed. However, there is a risk of infection with viruses and pathogens that have not yet been identified. An answer to this problem could be to strengthen the immunity, which could respond to infections by unknown viruses or pathogens $[3,4]$. To this end, many researchers are exploring materials with immune-enhancing properties and are studying the mechanisms behind these properties. For example, Panax ginseng, also called Korean red ginseng, has been extensively used in traditional medicine and as a functional food. It can boost the immune system by enhancing the phagocytic ability of macrophages, reinforcing the activity of natural killer cells, and inducing the production of mucosal secretory immunoglobulin (Ig) A [5-7]. Moreover, various immune-enhancing materials such as Aloe vera and propolis are being developed as health functional foods [8-11].

Probiotics (defined as live microorganisms) are known to mostly function by improving the intestinal environments 
through increasing the viability of beneficial bacteria and reducing the growth of harmful bacteria [12]. In addition, probiotics used as functional foods have been found to play an important role in controlling immune reactions, which has resulted in various health benefits being claimed, including enhancement of the immunity, control of hypersensitivity (allergy), and inhibition of inflammation [12-15]. The probiotic mixture VSL\#3 (Bifidobacterium breve, Bifidobacterium longum, Bifidobacterium infantis, Lactobacillus acidophilus, Lactobacillus plantarum, Lactobacillus paracasei, Lactobacillus bulgaricus, and Streptococcus thermophilus), for example, has been found to have multiple functions, and it has been indicated for the enhancement of the immune system, attenuation of allergic responses, and treatment of inflammatory bowel disease and irritable bowel syndrome [16-19].

Recently, many studies have reported that heat-killed microorganisms or fractionated cellular components, such as cytoplasmic extracts, membrane fragments, exopolysaccharides, and lipoteichoic acids, can modulate various immune responses, alleviating symptoms of colitis, autoimmune disorders, and allergies [20,21]. For this kind of substances, Mitsuoka [22] suggested the term "biogenics," which is defined as "food ingredients which beneficially affect the host by directly immunostimulating or suppressing mutagenesis, tumorigenesis, peroxidation, hypercholesterolemia, or intestinal putrefaction," and includes the administration of nonviable probiotic bacteria to obtain some "probiotic" effects [23]. Biogenic materials, including nonviable probiotic bacteria, have been actively developed as health supplements or functional foods because they possess stability to processes involving heat or acids [24, 25].

Nanometric Lactobacillus plantarum (strain name: nF1) (nLp-nF1) is a biogenics consisting of dead (nonviable) L. plantarum cells pretreated with heat and a nanodispersion process. The nLp-nF1 has a shrunken, granular shape and a size of $0.5-1.0 \mu \mathrm{m}$, and it has been registered in the DNA Data Bank of Japan (Accession No. NITE-P1462). In vitro studies using splenocytes of BALB/c mice have shown that nLp-nF1 has immunostimulating activities, involving the production of interleukin (IL)-12 and interferon (IFN)- $\alpha$ [26-28]. Furthermore, administration of nLp-nF1 effectively attenuates dextran sulfate sodium (DSS)-induced colitis symptoms and DSS-azoxymethane-induced colorectal cancer $[29,30]$. These anti-inflammatory and tumor suppressive effects might be derived from the induction of $\mathrm{T}$ helper (Th) 1 cells by IL-12, and from the induction of tumor necrosis factor-alpha (TNF- $\alpha$ ) production.
In the present study, we investigated whether nLp-nF1 enhances the immune response in primary splenocytes isolated from mice. Furthermore, we examined whether administration of nLp-nF1 has immunostimulating effects in the cyclophosphamide (CPP)-induced immunosuppressed mouse model. Finally, we assessed whether nLp-nF1 induces IL-12 production when used as a functional food ingredient in combination with various general foods.

\section{Materials and Methods}

\section{Materials}

To measure IL-12, TNF- $\alpha$, IL-1 $\beta$, and IFN- $\gamma$, enzyme-linked immunosorbent assay (ELISA) kits were purchased from BD Biosciences (USA), and an IgG ELISA kit was obtained from Santa Cruz Biotechnology (USA). Anti-CD3 (FITC), anti-CD11b (APC), anti-CD11c (PE), and anti-CD335 (APC/Fire 750) antibodies were purchased from Biolegend (USA). CPP was purchased from Tokyo Chemical Industry Co. (Japan), and concanavalin A (Con A) was obtained from Sigma-Aldrich (USA).

\section{Sample Preparation}

The nLp-nF1 (deposited at the NITE Biological Resource Center, Tokyo; Accession No. NITE-P1462) used in this study was obtained from Biogenics Korea Co., Ltd. (Korea). Briefly, nLp-nF1 was manufactured by incubating Lactobacillus plantarum for $20 \mathrm{~h}$ under $\mathrm{pH}$ control, followed by incubation at high temperature $\left(40^{\circ} \mathrm{C}\right)$, high salinity $(1.0 \%(\mathrm{w} / \mathrm{w}))$, and low $\mathrm{pH}(\mathrm{pH} 5.0)$ for $4 \mathrm{~h}$. Then, the sample was sterilized $\left(80^{\circ} \mathrm{C}, 10 \mathrm{~min}\right)$ and nanodispersed by highpressure homogenization $[27,28]$.

\section{Animals}

Five-week-old female BALB/c mice for in vitro experiments and 6-week-old male ICR mice for in vivo experiments were purchased from OrientBio Inc. (Korea). The animals were housed in an air-conditioned room $\left(23 \pm 2^{\circ} \mathrm{C}\right)$ with a $12 \mathrm{~h}$ light/dark cycle in accordance with the guidelines for animal use and care of the Korea Food Research Institute (Permit No. KFRI-M-16044).

In Vivo Experiments Using an Immunosuppressed Mouse Model ICR mice were divided into four groups consisting of naive, sham, low-dose $\mathrm{nLp}-\mathrm{nF} 1\left(4 \times 10^{9} \mathrm{CFU} / \mathrm{kg}\right.$ body weight), and highdose $\mathrm{nLp}-\mathrm{nF} 1\left(4 \times 10^{10} \mathrm{CFU} / \mathrm{kg}\right.$ body weight $)(n=5)$. Mice of all groups, except the naive group, were intraperitoneally injected with CCP $(150 \mathrm{mg} / \mathrm{kg} /$ day $)$ twice, once on day 0 and again on day 2, for induction of immunosuppression (Fig. 1). Mice in the $\mathrm{nLp}-\mathrm{nF} 1$ group were orally administered with nLp-nF1 once a day on days 3 to 17 (15 days). All mice were killed on day 18. For analysis of IgG levels, serum samples were obtained by collecting blood from the orbital venous plexus. Spleens and bone marrows were removed and were used for analysis of cytokine production. 


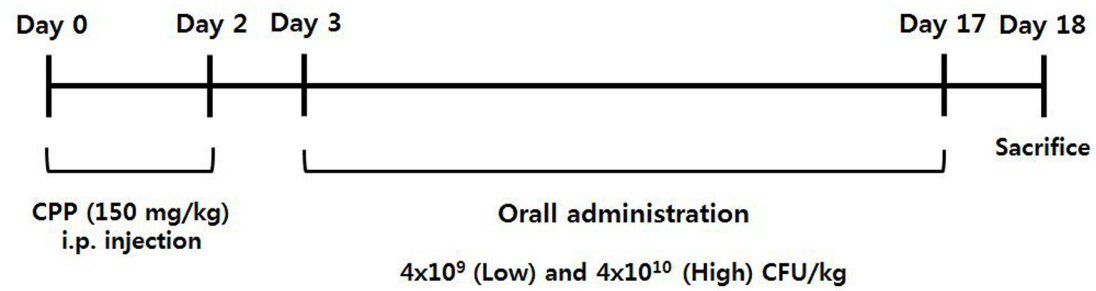

Fig. 1. Experimental schedule of the cyclophosphamide (CPP)-induced immunosuppressed mouse model.

\section{Culture of Splenocytes and Bone Marrow Cells}

Splenocytes were isolated from the spleens of ICR mice (immunosuppressive mouse model), and cells were seeded at $1 \times 10^{6}$ cell $/ \mathrm{ml}$ with Con A $(2 \mu \mathrm{g} / \mathrm{ml})$ in 12 -well plates. After $48 \mathrm{~h}$, the cell supernatants were harvested to measure cytokines levels.

Bone marrow cells were obtained from the long bones of ICR mice, and cells were seeded at $5 \times 10^{6} \mathrm{cell} / \mathrm{ml}$ in 12 -well plates in RPMI medium without Con A and containing 10\% fetal bovine serum (FBS). The cell supernatants were harvested after $72 \mathrm{~h}$ for evaluation of cytokine production.

\section{Measurement of Cytokines and Serum IgG Levels Using ELISA}

The levels of cytokines (IL-12, TNF- $\alpha$, and IFN- $\gamma$ ) and serum IgG were measured using ELISA kits according to the manufacturer's protocols. Briefly, recovered supernatants and standard solutions were transferred to 96-well plates precoated with the appropriate monoclonal antibodies against each of the target cytokines, and then incubated at room temperature for $2 \mathrm{~h}$. After thorough washing with buffer, horseradish peroxidase-conjugated secondary antibodies were added to each well, and incubation was continued at room temperature for $2 \mathrm{~h}$. After removal of the secondary antibody, the substrate for the enzymatic reaction was added, and samples were incubated for another $30 \mathrm{~min}$ in the dark. The reaction was terminated by addition of stop solution, and the absorbance was measured at $450 \mathrm{~nm}$ using a microplate reader (Molecular Devices, USA).

\section{Analysis of the Population of Bone Marrow Cells Using Flow Cytometry}

Bone marrow cells were gathered and suspended in Hank's balanced salt solution containing $10 \%$ FBS, $10 \%$ sodium azide, and $8 \%$ sodium bicarbonate. The cells were incubated on ice with $\mathrm{FC}_{\mathrm{C}}$ Block (anti-CD16/32) (BD Pharmagen, USA), and then they were stained with anti-CD3 (FITC), anti-CD11b (APC), anti-CD11c (PE), and anti-CD335 (APC/Fire 750) antibodies for $30 \mathrm{~min}$. The presence of single cells was confirmed by flow cytometry, which was performed using CytoFLEX (Beckman Coulter, USA).

\section{In Vitro Experiments Using Splenocytes}

Splenocytes were prepared by aseptically removing the spleens from BALB/c mice. The tissue was homogenized, and cells were collected and treated with red blood cell lysis buffer. Cell counting was performed by the trypan blue exclusion method, and the number of splenocytes was adjusted to a cell density of $5 \times 10^{6}$ cells $/ \mathrm{ml}$ in RPMI 1640 medium (10\% FBS). Splenocytes were then cultured in the absence or presence of nLp-nF1 at $37^{\circ} \mathrm{C}$ for up to $72 \mathrm{~h}$ in a humidified incubator with $5 \% \mathrm{CO}_{2}$. The culture supernatants were harvested and used to evaluate the effects of $\mathrm{nLp}-\mathrm{nF} 1$ on cytokine production by ELISA.

\section{Treatment of Products Combined with nLp-nF1}

For in vitro experiment of products combined with nLp-nF1, each product was diluted in PBS as follows: porridge, $1 / 20$; instant coffee, $1 / 20$; beverage, $1 / 20$; dry milk, $1 / 20$; sauce, $1 / 2,000$. Next, nLp-nF1 was added to the products at a dose of $5 \times 10^{7} \mathrm{CFU} / \mathrm{ml}$, and then splenocytes were treated with the products combined with nLp-nF1 in culture media. After $72 \mathrm{~h}$, the culture supernatants were harvested and used to evaluate the effects of nLp-nF1 on cytokine production by ELISA.

\section{Statistical Analysis}

Results are expressed as the mean \pm standard deviation (SD). Statistical analysis of the differences between experimental groups were assessed by one-way analysis of variance followed by Fprotected Fisher's least significant difference tests.

\section{Results and Discussion}

\section{nLp-nF1 Increases Serum IgG Levels in CPP-Induced Immunosuppressed Mice}

There are five classes of immunoglobulins: $\operatorname{IgA}, \operatorname{IgD}, \operatorname{IgE}$, $\operatorname{IgG}$, and IgM [31]. They have different roles in the immune response. IgG is the most common immunoglobulin in blood, lymph fluid, cerebrospinal fluid, and peritoneal fluid, and it is a key player in humoral immunity [32]. IgG can interact with macrophages, neutrophils, and natural killer cells, leading to activation of these cells [33]. In this study, we assessed serum IgG levels as an indicator of immune stimulation or immune enhancement. To investigate the immune-enhancing effect of $n L p-n F 1$, the immunosuppressed mouse model (CPP-induced) was used. $\mathrm{CPP}$ is an immunosuppressor used in neoplastic and severe autoimmune disorders because it reduces a wide variety of immune responses such as those from macrophages, 


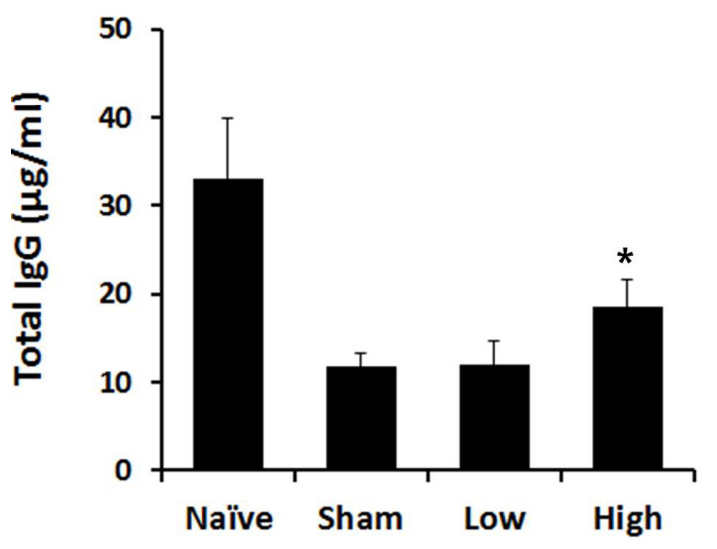

Fig. 2. Immune-enhancing effect of $n L p-n F 1$ on serum $\operatorname{IgG}$ levels in cyclophosphamide (CPP)-induced immunosuppressed ICR mice.

Serum from blood of the orbital venous plexus of CPP-treated mice was used to measure total IgG levels by ELISA. Data are presented as the mean \pm SD. Asterisks $\left(^{*}\right)$ indicate significant differences $(p<0.05)$ between the sham and nLp-nF1 groups.

neutrophils, and effector T cells [34-36]. As expected, the CPP-injected group had reduced levels of IgG in sera compared with the control group, whereas the IgG levels in the group orally administered with nLp-nF1 were significantly higher than that in the CPP-treated group (Fig. 2). In general, an increase of IgG levels is able to connect with Th1-dominant immune responses, and the immune system maintains immune balance with Th1- and Th2-related immune responses [37]. Thus, we additionally investigated the levels of serum $\operatorname{IgE}$ as a Th2-mediated main response. As a result, the serum $\operatorname{IgE}$ was generally detected at a very low level, and the administration of nLpnF1 could not change the IgE levels (data not shown). The result demonstrates that administration of nLp-nF1 can contribute to stimulation of the systemic immune system, leading to immune enhancement by increasing IgG levels (Th1-mediated immune response) without the regulation of IgE levels (Th2-mediated immune response).

\section{nLP-nF1 Induces Production of IL-12 and TNF- $\alpha$ in Bone} Marrow Cells from CPP-Induced Immunosuppressed Mice

The bone marrow is a primary immune organ that is important in the production of B lymphocytes and the precursor of $\mathrm{T}$ lymphocytes. Furthermore, in the bone marrow, immature myeloid cells are generated and differentiated to granulocytes, monocytes, macrophages, natural killer cells, and dendritic cells [38, 39]. Macrophages, natural killer cells, and dendritic cells originated in the
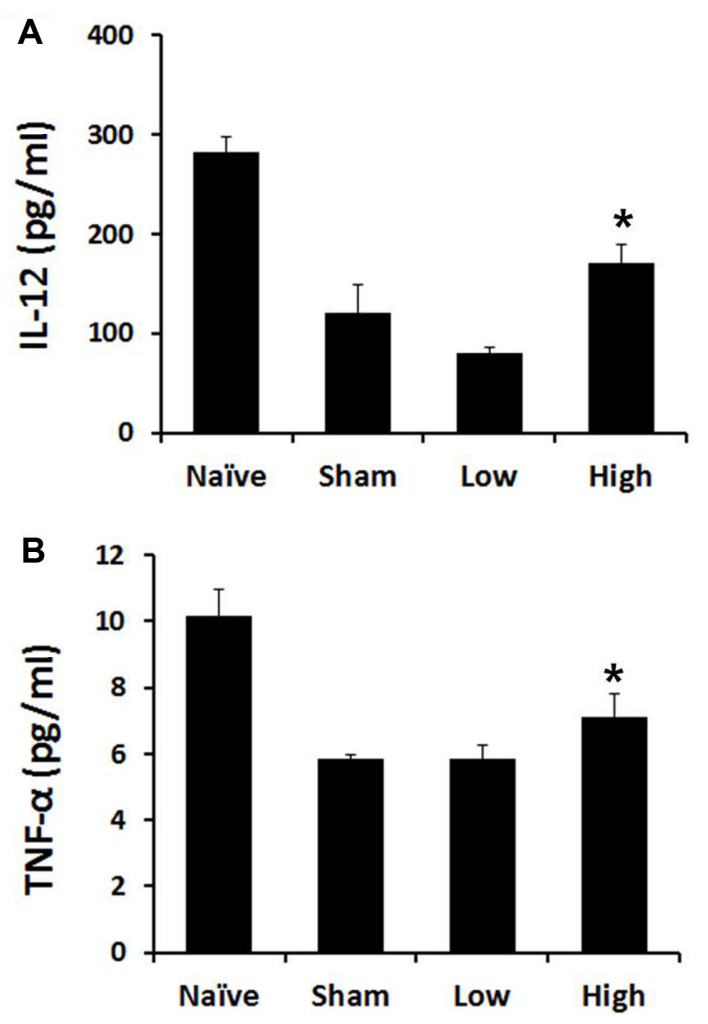

Fig. 3. Immune-enhancing effect of $n L p-n F 1$ on cytokine production by bone marrow cells isolated from cyclophosphamideinduced immunosuppressed ICR mice.

Bone marrow cells were cultured for $72 \mathrm{~h}$, and production of the cytokines (A) IL-12 and (B) TNF- $\alpha$ in culture supernatants was measured by ELISA. Data are presented as the mean \pm SD of three independent experiments. Asterisks $\left(^{*}\right)$ indicate significant differences $(p<0.05)$ between the sham and nLp-nF1 groups.

bone marrow play a key role in enhancing or regulating the innate immunity. Therefore, we investigated whether administration of $\mathrm{nLp}-\mathrm{nF} 1$ induces the production of immune-enhancing cytokines such as IL-12 and TNF- $\alpha$ by bone marrow cells isolated from CPP-induced immunosuppressed mice. As expected, the CPP-injected group showed a reduced production of IL-12 and TNF- $\alpha$ by bone marrow cells compared with the naive group. However, the reduced production of cytokines was significantly recovered by oral administration of high-dose nLp-nF1 (Fig. 3). In bone marrow, IL-12 can be produced from monocytes, macrophages, and dendritic cells, whereas TNF- $\alpha$ can be produced by monocytes, macrophages, dendritic cells, lymphoid cells, and natural killer cells [40, 41]. To examine which of these cells was responsible for IL-12 and TNF- $\alpha$ production in our model, the population of immune cells in bone marrow, including $\mathrm{CD}^{+}$cells, 

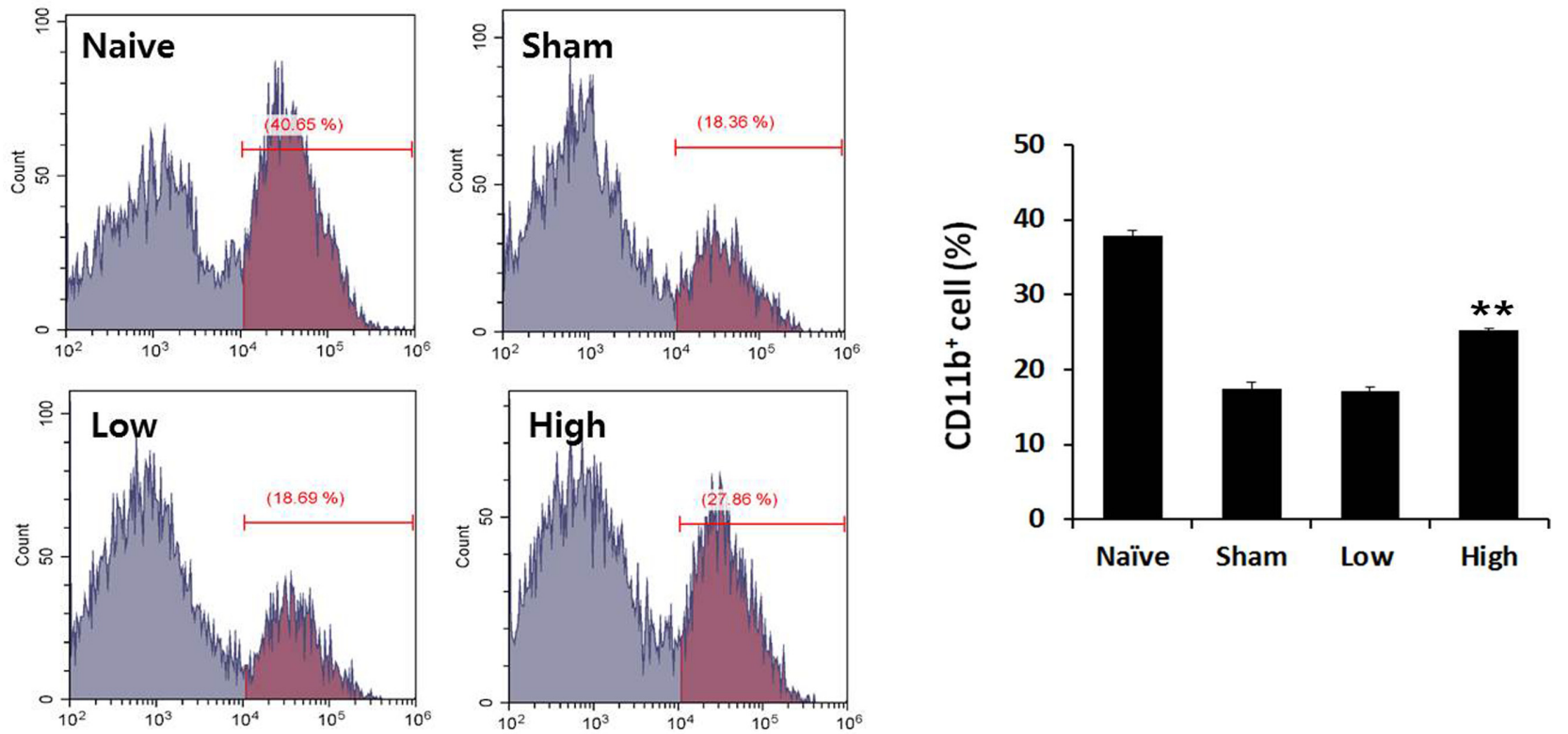

Fig. 4. Effect of orally administered $\mathrm{nLp}-\mathrm{nF} 1$ on the population of $\mathrm{CD}_{11} \mathrm{~b}^{+}$cells in bone marrow cells isolated from cyclophosphamide-induced immunosuppressed ICR mice.

Bone marrow $\mathrm{CD}_{11 \mathrm{~b}^{+}}$cells were stained with anti-CD11b and were analyzed by flow cytometry. Data are presented as the mean $\pm \mathrm{SD}$ of three independent experiments. Asterisks $(* *)$ indicate significant differences $(p<0.01)$ between the sham and nLp-nF1 groups.

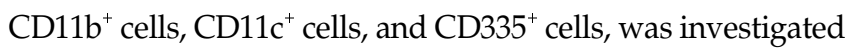
by flow cytometry. We found that high-dose administration of $n L p-n F 1$ resulted in an increase in the $\mathrm{CD}_{11 b^{+}}$cell population compared with the CPP-injected group (Fig. 4), but no changes in the $\mathrm{CD}^{+}$cell, CD11 $\mathrm{c}^{+}$cell, and $\mathrm{CD} 335^{+}$ cell populations were detected (data not shown). In bone marrow, there are many kinds of CD11b-expressing cells such as monocytes, macrophages, dendritic cells, and granulocytes, and the proportion of $\mathrm{CD}_{11 b^{+}}$cells accounts for $44 \%$ of bone marrow cells $[42,43]$. The cells have been known to be able to produce IL-12 and TNF- $\alpha$ as stimulatory factors $[44,45]$. These stimulatory factors are able to activate immune cells through autocrine and paracrine signaling, resulting in an increase of proliferation or induction of differentiation. In the present study, Figs. 3 and 4 show that IL-12 and TNF- $\alpha$ were increased by nLp-nF1, and only the population of $\mathrm{CD}_{11 \mathrm{~b}^{+}}$cells was increased. Therefore, we suggest that nLp-nF1 might activate the IL-12- and TNF- $\alpha$-producing cells and then the produced stimulatory factors activate the $\mathrm{CD} 11 \mathrm{~b}^{+}$cells, resulting in an increase in both the cell population and cytokine levels. These results indicate that $\mathrm{nLp}-\mathrm{nF} 1$ administration enhances the immune system by inducing IL-12 and TNF- $\alpha$ production by $\mathrm{CD}_{11} \mathrm{~b}^{+}$cells in bone marrow.
nLP-nF1 Increases the Production of IL-12, TNF- $\alpha$, and IFN- $\gamma$ in Splenocytes Isolated from CPP-Induced Immunosuppressed Mice

The spleen is the largest secondary immune organ in the body, and it has a filtering function for circulating blood. It is also very important for the maintenance of immune homeostasis, affecting both the innate and the adaptive arms of the systemic immune system [46, 47]. Therefore, we investigated the effect of $\mathrm{nLp}-\mathrm{nF} 1$ on splenocytes isolated from CPP-induced immunosuppressed mice. Administration of high-dose nLp-nF1 strongly increased the production of IL-12 and TNF- $\alpha$ by splenocytes (Figs. 5A and $5 \mathrm{~B})$. IL-12 and TNF- $\alpha$ produced by innate immune cells lead to activation of adaptive immune cells, such as CD4 ${ }^{+}$ and $\mathrm{CD}^{+} \mathrm{T}$ cells, resulting in the production of IFN- $\gamma$. Therefore, we measured the levels of IFN- $\gamma$ to assess the activation of the adaptive immunity. We found that IFN- $\gamma$ levels were significantly enhanced by the administration of high-dose nLp-nF1 (Fig. 5C). These results demonstrate that administration of high-dose nLp-nF1 can enhance not only the innate immunity (IL-12 and TNF- $\alpha$ ) but also the adaptive immunity (IFN- $\gamma$ ). In this study, therefore, we suggest that the enhancing effects of $n L p-n F 1$ have been verified in both arms of the systemic immune system. 

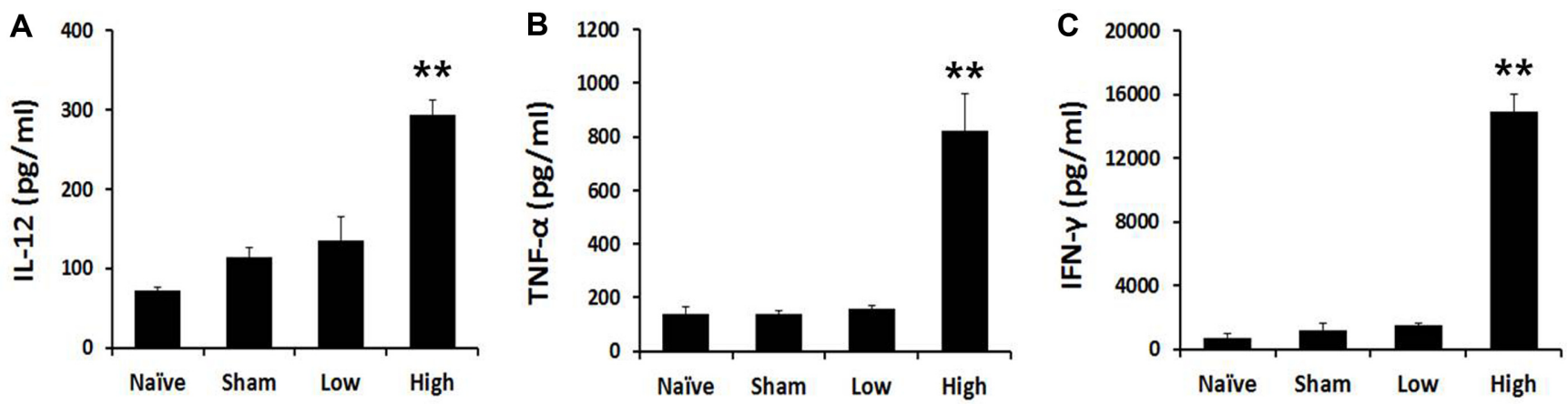

Fig. 5. Immune-enhancing effect of nLp-nF1 on cytokine production by splenocytes isolated from cyclophosphamide-induced immunosuppressed ICR mice.

Splenocytes were cultured with Con A ( $2 \mu \mathrm{g} / \mathrm{ml})$ for $48 \mathrm{~h}$, and production of (A) IL-12, (B) TNF- $\alpha$, and (C) IFN- $\gamma$ was measured in the culture supernatants by ELISA. Data are presented as the mean \pm SD of three independent experiments. Asterisks $(* *)$ indicate significant differences $(p<0.01)$ between the sham and nLp-nF1 groups.

Interestingly, we found a difference between low-dose and high-dose administration of nLp-nF1 on immune enhancement in our CCP-induced immune suppressive mouse model. The administration of low-dose nLp-nF1 could not enhance the IgG, IL-12, TNF- $\alpha$, and IFN- $\gamma$ levels. We thought that since the immunosuppression induced by CCP was too strong, the immune enhancing effect of nLp$\mathrm{nF} 1$ at low dose did not seem to be effective in this model. Nevertheless, the administration of high-dose nLp-nF1 strongly increased the levels of immunoglobulin and cytokines. We suggest that the high-dose nLp-nF1 can be effective as an immune enhancer, where the remarkable effect of $\mathrm{nLp}-\mathrm{nF} 1$ may be derived from its nanometric property.

Enhancing Effect of nLp-nF1 on the Immune Response in an In Vitro System

In the present study, we have shown that oral administration of nLp-nF1 can contribute to the enhancement of the immune response, including both humoral and cellular immunities (innate and adaptive immunities). Unlike probiotics (live microorganisms), since nLp-nF1 is a heatkilled microorganism, its properties can resist the heat and acid treatments of various processes in the manufacturing of end products. Therefore, we examined whether treatment with nLp-nF1 or with other materials, on their own or in combination with nLp-nF1, enhances the immune response in an in vitro system.

First, we investigated the effects of nLp-nF1 on whole splenocytes isolated from mice. We found that production of the cytokines IL-12, TNF- $\alpha$, and IFN- $\gamma$ increased significantly with $\mathrm{nLp}-\mathrm{nF} 1$ treatment in a dose-dependent manner (Fig. 6). This result indicates that nLp-nF1 treatment could enhance the immune response in both in vitro and in vivo systems.

Next, we examined the enhancing effects of various
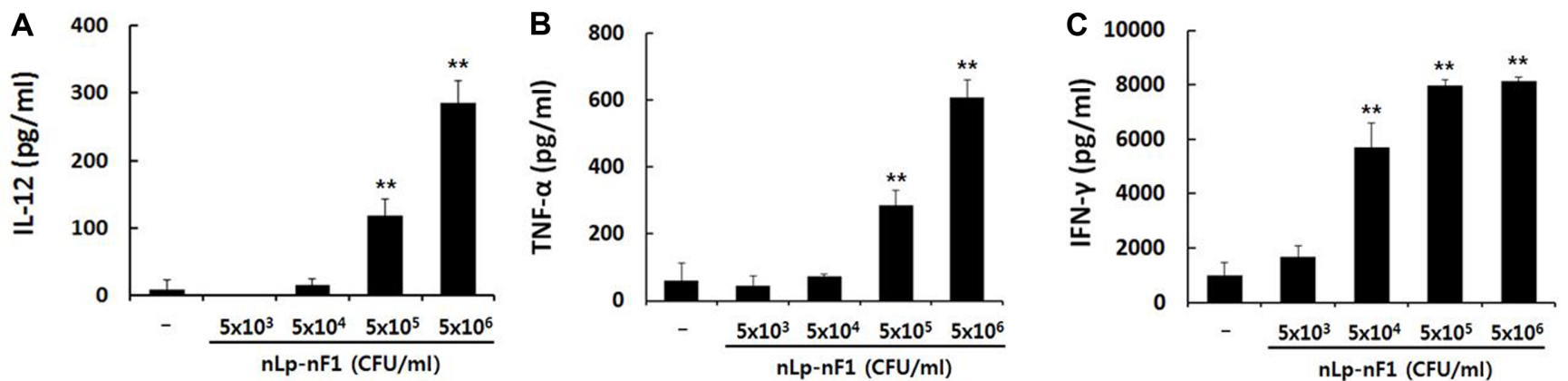

Fig. 6. Immune-enhancing effect of nLp-nF1 on cytokine production by splenocytes in vitro.

Splenocytes were cultured with or without nLp-nF1 for $72 \mathrm{~h}$ and production of (A) IL-12, (B) TNF- $\alpha$, and (C) IFN- $\gamma$ was measured in the culture supernatants by ELISA. Data are presented as the mean \pm SD of three independent experiments. Asterisks $\left.{ }^{* *}\right)$ indicate significant differences $(p<0.01)$. 

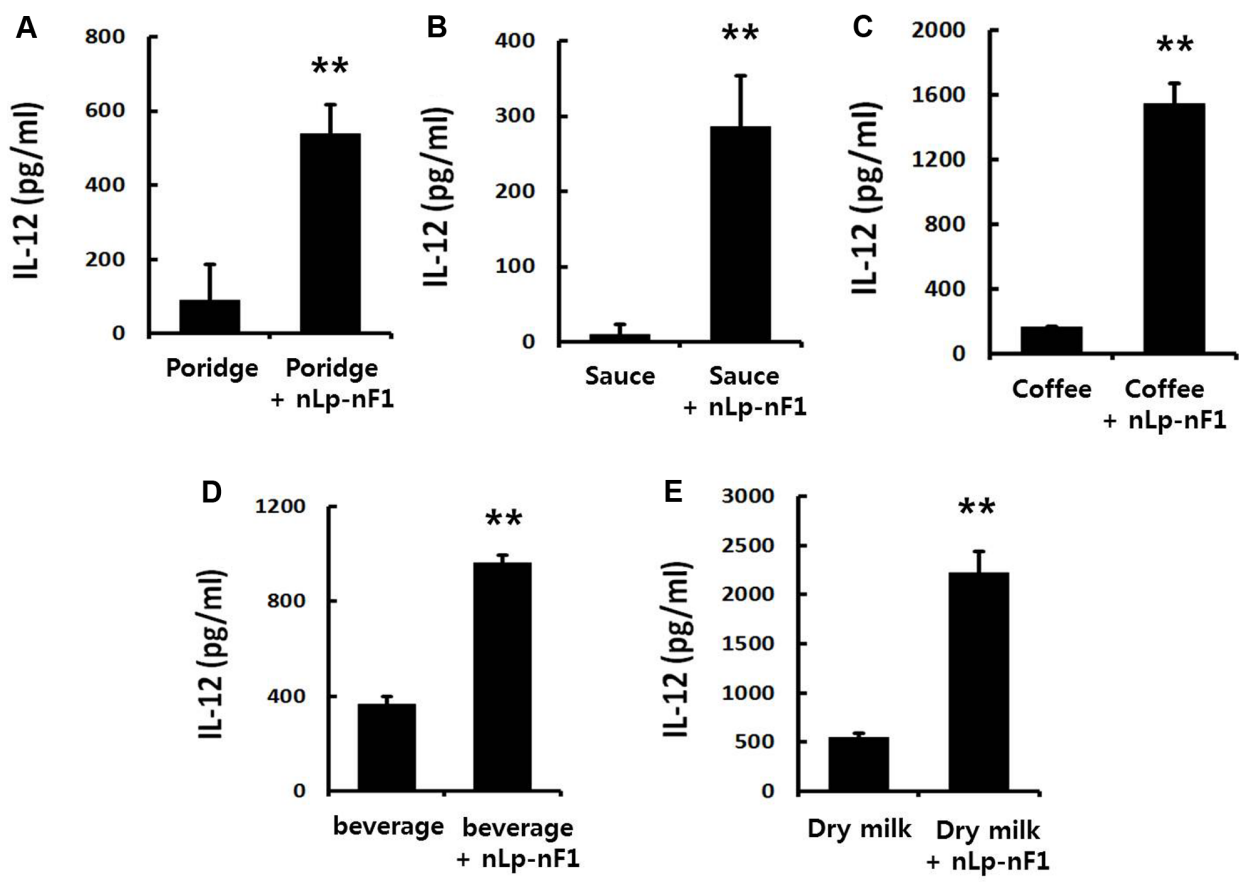

Fig. 7. Effects of food materials in combination with nLp-nF1 on the production of the cytokine IL-12.

Splenocytes were cultured with various food materials on their own or in combination with nLp-nF1 for $72 \mathrm{~h}$. IL-12 cytokine levels were measured by ELISA in the culture supernatants of cells treated with (A) nLp-nF1 plus porridge, (B) nLp-nF1 plus sauce, (C) nLp-nF1 plus coffee, (D) nLp$\mathrm{nF} 1$ plus beverage, and (E) nLp-nF1 plus dry milk. Data are presented as the mean \pm SD of three independent experiments. Asterisks $(* *)$ indicate significant differences $(p<0.01)$ between treatments with and without nLp-nF1.

products (porridge, sauce, instant coffee, beverage, and dry milk) on their own or in combination with $5 \times 10^{7} \mathrm{CFU} / \mathrm{ml}$ of nLp-nF1. All products when combined with nLp-nF1 induced the production of IL-12 in vitro system (Fig. 7). This result demonstrates that nLp-nF1 can enhance the immune response by inducing IL-12, TNF- $\alpha$, and IFN- $\gamma$ production even in combination with a variety of products. This suggests that $\mathrm{nLp}-\mathrm{nF} 1$ used as a food additive would be able to give the functionalities of nLp-nF1 to general foods.

In conclusion, we have shown that nanometric L. plantarum (nLp-nF1) has immune-enhancing effects both in vitro and in vivo. This suggests that uptake of nLp-nF1 could contribute to enhancement of the immune system and to the recovery of normal levels of immunity in people with reduced immunity, such as children, the elderly, and patients. Furthermore, $\mathrm{nLp}-\mathrm{nF} 1$ could be used as a functional food, a probiotic drug, and a functional food additive to stimulate the immune response.

\section{Acknowledgments}

This work was supported by the Korea Institute for
Planning and Evaluation for Technology in Food (iPET) through the High Value-added Food Technology Development Program, funded by the Ministry of Agriculture, Food and Rural Affaires (No. 314073-03) and by a research grant (Grant No. E0170401-01) from the Korea Food Research Institute.

\section{Conflict of Interest}

The authors have no financial conflicts of interest to declare.

\section{References}

1. Schiavoni G, D'Amato G, Afferni C. 2017. The dangerous liaison between pollens and pollution in respiratory allergy. Ann. Allergy Asthma Immunol. 118: 269-275.

2. LaKind JS, Overpeck J, Breysse PN, Backer L, Richardson SD, Sobus J, et al. 2016. Exposure science in an age of rapidly changing climate: challenges and opportunities. J. Expo. Sci. Environ. Epidemiol. 26: 529-538.

3. Cesarone MR, Belcaro G, Di Renzo A, Dugall M, Cacchio M, Ruffini I, et al. 2007. Prevention of influenza episodes with colostrum compared with vaccination in healthy and high- 
risk cardiovascular subjects: the epidemiologic study in San Valentino. Clin. Appl. Thromb. Hemost. 13: 130-136.

4. Xu ML, Kim HJ, Chang DY, Kim HJ. 2013. The effect of dietary intake of the acidic protein fraction of bovine colostrum on influenza A (H1N1) virus infection. J. Microbiol. 51: 389-393.

5. Park HY, Lee SH, Lee KS, Yoon HK, Yoo YC, Lee J, et al. 2015. Ginsenoside Rg1 and 20(S)-Rg3 induce IgA production by mouse B cells. Immune Netw. 15: 331-336.

6. Du XF, Jiang CZ, Wu CF, Won EK, Choung SY. 2008. Synergistic immunostimulatory effect of pidotimod and red ginseng acidic polysaccharide on humoral immunity of immunosuppressed mice. Pharmazie 63: 904-908.

7. Byeon SE, Lee J, Kim JH, Yang WS, Kwak YS, Kim SY, et al. 2012. Molecular mechanism of macrophage activation by red ginseng acidic polysaccharide from Korean red ginseng. Mediators Inflamm. 2012: 732860.

8. Choi S, Chung M-H. 2003. A review on the relationship between aloe vera components and their biologic effects. Semin. Integr. Med. 1: 53-62.

9. Christaki EV, Florou-Paneri PC. 2010. Aloe vera: a plant for many uses. J. Food Agric. Environ. 8: 245-249.

10. Sforcin JM. 2007. Propolis and the immune system: a review. J. Ethnopharmacol. 113: 1-14.

11. Orsolic N, Sver L, Terzic S, Basic I. 2005. Peroral application of water-soluble derivative of propolis (WSDP) and its related polyphenolic compounds and their influence on immunological and antitumor activity. Vet. Res. Commun. 29: 575-593.

12. Lane ER, Zisman TL, Suskind DL. 2017. The microbiota in inflammatory bowel disease: current and therapeutic insights. J. Inflamm. Res. 10: 63-73.

13. D'Angelo C, Reale M, Costantini E. 2017. Microbiota and probiotics in health and HIV infection. Nutrients 9: 1-15.

14. McKenzie C, Tan J, Macia L, Mackay CR. 2017. The nutritiongut microbiome-physiology axis and allergic diseases. Immunol. Rev. 278: 277-295

15. Aitoro R, Paparo L, Amoroso A, Di Costanzo M, Cosenza L, Granata V, et al. 2017. Gut microbiota as a target for preventive and therapeutic intervention against food allergy. Nutrients 9: 1-12.

16. Manuzak JA, Hensley-McBain T, Zevin AS, Miller C, Cubas R, Agricola B, et al. 2016. Enhancement of microbiota in healthy macaques results in beneficial modulation of mucosal and systemic immune function. J. Immunol. 196: 2401-2409.

17. Schiavi E, Barletta B, Butteroni C, Corinti S, Boirivant M, Di Felice G. 2011. Oral therapeutic administration of a probiotic mixture suppresses established Th2 responses and systemic anaphylaxis in a murine model of food allergy. Allergy 66: 499-508.

18. Distrutti E, Cipriani S, Mencarelli A, Renga B, Fiorucci S. 2013. Probiotics VSL\#3 protect against development of visceral pain in murine model of irritable bowel syndrome. PLoS One 8: e63893.
19. Isidro RA, Lopez A, Cruz ML, Gonzalez Torres MI, Chompre G, Isidro AA, et al. 2017. The probiotic VSL\#3 modulates colonic macrophages, inflammation, and microflora in acute trinitrobenzene sulfonic acid colitis. J. Histochem. Cytochem. 65: 445-461.

20. Adams CA. 2010. The probiotic paradox: live and dead cells are biological response modifiers. Nutr. Res. Rev. 23: 37-46.

21. De Vries MC, Vaughan EE, Kleerebezem M, De Vos WM. 2006. Lactobacillus plantarum survival, functional and potential probiotic properties in the human intestinal tract. Int. Dairy J. 16: 1018-1028.

22. Mitsuoka T. 2000. Significance of dietary modulation of intestinal flora and intestinal environment. Biosci. Microflora 19: $15-25$.

23. Ohshima T, Kojima Y, Seneviratne CJ, Maeda N. 2016. Therapeutic application of synbiotics, a fusion of probiotics and prebiotics, and biogenics as a new concept for oral Candida infections: a mini review. Front. Microbiol. 7: 10.

24. Terada A, Bukawa W, Kan T, Mitsuoka T. 2004. Effects of the consumption of heat-killed Enterococcus faecalis EC-12 preparation on microbiota and metabolic activity of the faeces in healthy adults. Microbial Ecol. Health Dis. 16: 188-194.

25. Sawada D, Sugawara T, Ishida Y, Aihara K, Aoki Y, Takehara I, et al. 2016. Effect of continuous ingestion of a beverage prepared with Lactobacillus gasseri CP2305 inactivated by heat treatment on the regulation of intestinal function. Food Res. Int. 79: 33-39.

26. Hasegawa H, Kan T. 2008. Immunity for longevity and lactic acid bacteria: the effect of nanometric particles of lactic acid bacteria on Th1 cell induction. New Food Ind. 50: 1-8.

27. Kan T, Ohwaki M. 2014. Lactobacillus having ability to induce IL-12 production, and method for culturing same. WO Patent, 2014/ 088183.

28. Lee HA, Kim H, Lee KW, Park KY. 2016. Dead Lactobacillus plantarum stimulates and skews immune responses toward T helper 1 and 17 polarizations in RAW 264.7 cells and mouse splenocytes. J. Microbiol. Biotechnol. 26: 469-476.

29. Lee HA, Kim H, Lee KW, Park KY. 2015. Dead nano-sized Lactobacillus plantarum inhibits azoxymethane/dextran sulfate sodium-induced colon cancer in Balb/c mice. J. Med. Food 18: 1400-1405.

30. Lee HA, Bong YJ, Kim H, Jeong JK, Kim HY, Lee KW, et al. 2015. Effect of nanometric Lactobacillus plantarum in kimchi on dextran sulfate sodium-induced colitis in mice. J. Med. Food. 18: 1073-1080.

31. Woof J, Burton D. 2004. Human antibody-Fc receptor interactions illuminated by crystal structures. Nat. Rev. Immunol. 4: 89-99.

32. Pier GB, Lyczak JB, Wetzler LM. 2004. Imunology, Infection, and Immunity. ASM Press, Washington, D.C.

33. Mallery DL, McEwan WA, Bidgood SR, Towers GJ, Johnson CM, James LC. 2010. Antibodies mediate intracellular immunity through tripartite motif-containing 21 (TRIM21). Proc. Natl. 
Acad. Sci. USA 107: 19985-19990.

34. Jin R, Wan LL, Mitsuishi T, Sato S, Akuzawa Y, Kodama K, et al. 1994. Effect of shi-ka-ron and Chinese herbs on cytokine production of macrophage in immunocompromised mice. Am. J. Chin. Med. 22: 255-266.

35. Zuluaga AF, Salazar BE, Rodriguez CA, Zapata AX, Agudelo M, Vesga O. 2006. Neutropenia induced in outbred mice by a simplified low-dose cyclophosphamide regimen: characterization and applicability to diverse experimental models of infectious diseases. BMC Infect. Dis. 6: 1-10.

36. Yasunami R, Bach JF. 1988. Anti-suppressor effect of cyclophosphamide on the development of spontaneous diabetes in NOD mice. Eur. J. Immunol. 18: 481-484.

37. Smith KM, Pottage L, Thomas ER, Leishman AJ, Doig TN, $\mathrm{Xu} \mathrm{D}$, et al. 2000. Th1 and Th2 $\mathrm{CD}^{+} \mathrm{T}$ cells provide help for B cell clonal expansion and antibody synthesis in a similar manner in vivo. J. Immunol. 165: 3136-3144.

38. Birbrair A, Frenette PS. 2016. Niche heterogeneity in the bone marrow. Ann. NY Acad. Sci. 1370: 82-96.

39. Vunjak-Novakovic G, Tandon N, Godier A, Maidhof R, Marsano A, Martens TP, et al. 2010. Challenges in cardiac tissue engineering. Tissue Eng. Part B Rev. 16: 169-187.

40. Kaushik RS, Uzonna JE, Zhang Y, Gordon JR, Tabel H. 2000. Innate resistance to experimental African trypanosomiasis: differences in cytokine (TNF-alpha, IL-6, IL-10 and IL-12) production by bone marrow-derived macrophages from resistant and susceptible mice. Cytokine 12: 1024-1034.

41. Wang C, Yu X, Cao Q, Wang Y, Zheng G, Tan TK, et al. 2013. Characterization of murine macrophages from bone marrow, spleen and peritoneum. BMC Immunol. 14: 6 .

42. Zhao E, Xu H, Wang L, Kryczek I, Wu K, Hu Y, et al. 2012. Bone marrow and the control of immunity. Cell. Mol. Immunol. 9: 11-19.

43. Springer TA. 1980. Cell-surface differentiation in the mouse, pp. 185-217. In Kennett RH, McKearn TJ, Bechtol KB (eds.), Monoclonal Antibodies. Springer, Boston, MA.

44. Ma X, Yan W, Zheng H, Du Q, Zhang L, Ban Y, et al. 2015. Regulation of IL-10 and IL-12 production and function in macrophages and dendritic cells. F1000Res 4: 1465.

45. Kruglov AA, Lampropoulou V, Fillatreau S, Nedospasov SA. 2011. Pathogenic and protective functions of TNF in neuroinflammation are defined by its expression in $\mathrm{T}$ lymphocytes and myeloid cells. J. Immunol. 187: 5660-5670.

46. Swirski FK, Nahrendorf M, Etzrodt M, Wildgruber M, Cortez-Retamozo V, Panizzi P, et al. 2009. Identification of splenic reservoir monocytes and their deployment to inflammatory sites. Science 325: 612-616.

47. Jia T, Pamer EG. 2009. Immunology. Dispensable but not irrelevant. Science 325: 549-550. 